\title{
Robust biomarker discovery for hepatocellular carcinoma from high-throughput data by multiple feature selection methods
}

\author{
Zishuang Zhang ${ }^{1}$ and Zhi-Ping Liu ${ }^{1,2^{*}}$ (1)
}

From Fifteenth International Conference on Intelligent Computing (ICIC 2019) Nanchang, China. 3-6 August 2019

\begin{abstract}
Background: Hepatocellular carcinoma (HCC) is one of the most common cancers. The discovery of specific genes severing as biomarkers is of paramount significance for cancer diagnosis and prognosis. The high-throughput omics data generated by the cancer genome atlas (TCGA) consortium provides a valuable resource for the discovery of HCC biomarker genes. Numerous methods have been proposed to select cancer biomarkers. However, these methods have not investigated the robustness of identification with different feature selection techniques.

Methods: We use six different recursive feature elimination methods to select the gene signiatures of HCC from TCGA liver cancer data. The genes shared in the six selected subsets are proposed as robust biomarkers. Akaike information criterion (AIC) is employed to explain the optimization process of feature selection, which provides a statistical interpretation for the feature selection in machine learning methods. And we use several methods to validate the screened biomarkers.
\end{abstract}

Results: In this paper, we propose a robust method for discovering biomarker genes for HCC from gene expression data. Specifically, we implement recursive feature elimination cross-validation (RFE-CV) methods based on six different classication algorithms. The overlaps in the discovered gene sets via different methods are referred as the identified biomarkers. We give an interpretation of the feature selection process based on machine learning using AIC in statistics. Furthermore, the features selected by the backward logistic stepwise regression via AIC minimum theory are completely contained in the identified biomarkers. Through the classification results, the superiority of interpretable robust biomarker discovery method is verified.

Conclusions: It is found that overlaps among gene subsets contain different quantitative features selected by the RFE-CV of 6 classifiers. The AIC values in the model selection provide a theoretical foundation for the feature selection process of biomarker discovery via machine learning. What's more, genes containing in more optimally selected

\footnotetext{
*Correspondence: zpliu@sdu.edu.cn

${ }^{1}$ Department of Biomedical Engineering, School of Control Science

and Engineering, Shandong University, Jinan 250061, Shandong, China

Full list of author information is available at the end of the article
} original author(s) and the source, provide a link to the Creative Commons licence, and indicate if changes were made. The images or other third party material in this article are included in the article's Creative Commons licence, unless indicated otherwise in a credit line to the material. If material is not included in the article's Creative Commons licence and your intended use is not permitted by statutory regulation or exceeds the permitted use, you will need to obtain permission directly from the copyright holder. To view a copy of this licence, visit http://creativecommons.org/licenses/by/4.0/. The Creative Commons Public Domain Dedication waiver (http://creativeco mmons.org/publicdomain/zero/1.0/) applies to the data made available in this article, unless otherwise stated in a credit line to the data. 
subsets make better biological sense and implication. The quality of feature selection is improved by the intersections of biomarkers selected from different classifiers. This is a general method suitable for screening biomarkers of complex diseases from high-throughput data.

Keywords: Biomarker discovery, Omics data, Feature selection, Akaike information criterion, Hepatocellular carcinoma

\section{Background}

The number of cancer deaths worldwide indicates HCC is the second leading cause in recent years [1]. Despite the practice of surveillance program, most of HCC patients are often examined in advanced stage [2]. Studies have shown liver cancer patients can significantly benefit from early screening [3]. Using effective molecular biomarkers is one of the most efficient way of realizing early cancer diagnosis. The availability of high-throughput omics data provides unprecedented opportunity and challenge for discoverying diagnostic biomarkers for HCC. For instance, the cohort study of TCGA provides amount of valuable data resources for the searching of cancer biomarkers [4].

So far, a number of methods of feature selection have been proposed to identify biomarkers from highthroughput data [5]. Joint with machine-learning-based classification algorithms, feature selection is a very useful strategy for biomarker discovery from ultra-dimensional omics data [6]. Usually, different feature selection methods may produce different feature ranking. Due to the high dimensionality of omics data, feature rankings have more possibilities [7]. Therefore, it is of great significance to realize the reproducibility of biomarkers and the robustness of biomarker discovery. Among the current feature selection methods, more attention is paid to whether the selected biomarkers can achieve a good classification performance. For example, the reference [8] has compared the classification performance of 10 kinds of machine learning algorithms. The applications of 6 kinds of machine learning methods for omics data have been implemented previously [9]. In addition, for the feature selection method of TCGA data, the reference [10] used network smoothing technology combined with PCA to select features. The reference [11] combined multiple levels of TCGA data to find key regulators and pathways between normal and tumor samples. A comprehensive feature selection strategy based on fuzzy rules has been experimented on TCGA data [12]. In order to realize the stability and reproducibility of biomarkers, a method combining individual signatures was proposed to improve the stability of feature selection [7]. However, the stability and reproducibility of biomarkers are still needed to be emphasized and strengthened in biomarker identification.
In this paper, we propose a robust method for discovering biomarker genes from transcriptomic data. Specifically, we implement RFE-CV methods based on 6 different classification algorithms, i.e., Adaboost, K-nearest neighbor (KNN), naïve Bayes (NB), neural network $(\mathrm{NN})$, random forest (RF) and support vector machine (SVM). We find their intersections by comparing the subsets of features selected by different classifiers. The repeatability and stability of biomarker discovery can be achieved by using the genes in the overlapping part. Feature selection by machine learning is often regarded as a black-box predictive model. In Tansey's work, the holdout randomization test is proposed to explain the black box statistically [13]. As we all known, Akaike information criterion (AIC) is very widely used in the selection of statistical models [14]. Inspired by the studies of Tansey and Akaike, we propose to explain RFE-CV with AIC in statistics. For shedding light to the black box of feature selection, we also introduce backward logistic stepwise regression for comparing and verifying the machinelearning-based feature selection process.

\section{Methods}

Figure 1 illustrates the framework of identifying cancer biomarkers from TCGA Liver Hepatocellular Carcinoma (LIHC) transcriptomic data, in which the four specific steps are contained.

\section{Data preprocessing}

We download the raw RNA sequencing (RNA-seq) data of HCC from Genomic Data Commons (GDC) using TCGA-Assembler 2.0 [15]. TCGA has sequenced more than 30 types of cancer and aims to provide a valuable data resource for the discovery of biomarkers [4]. For a proof-of-concept study, we use the RNA-seq data of HCC from the TCGA database which contains 20,530 genes and 423 samples, including 50 normal samples and 373 tumor samples. To achieve a balanced dataset in machine learning, we select 50 positive samples and their corresponding 50 negative samples. In other words, a pair of positive and negative samples are from the same donor's cancerous tissue and adjacent tissue respectively. We normalize the raw RNA-seq data using the median of ratios rule of DESeq2 [16] and remove the batch effects 


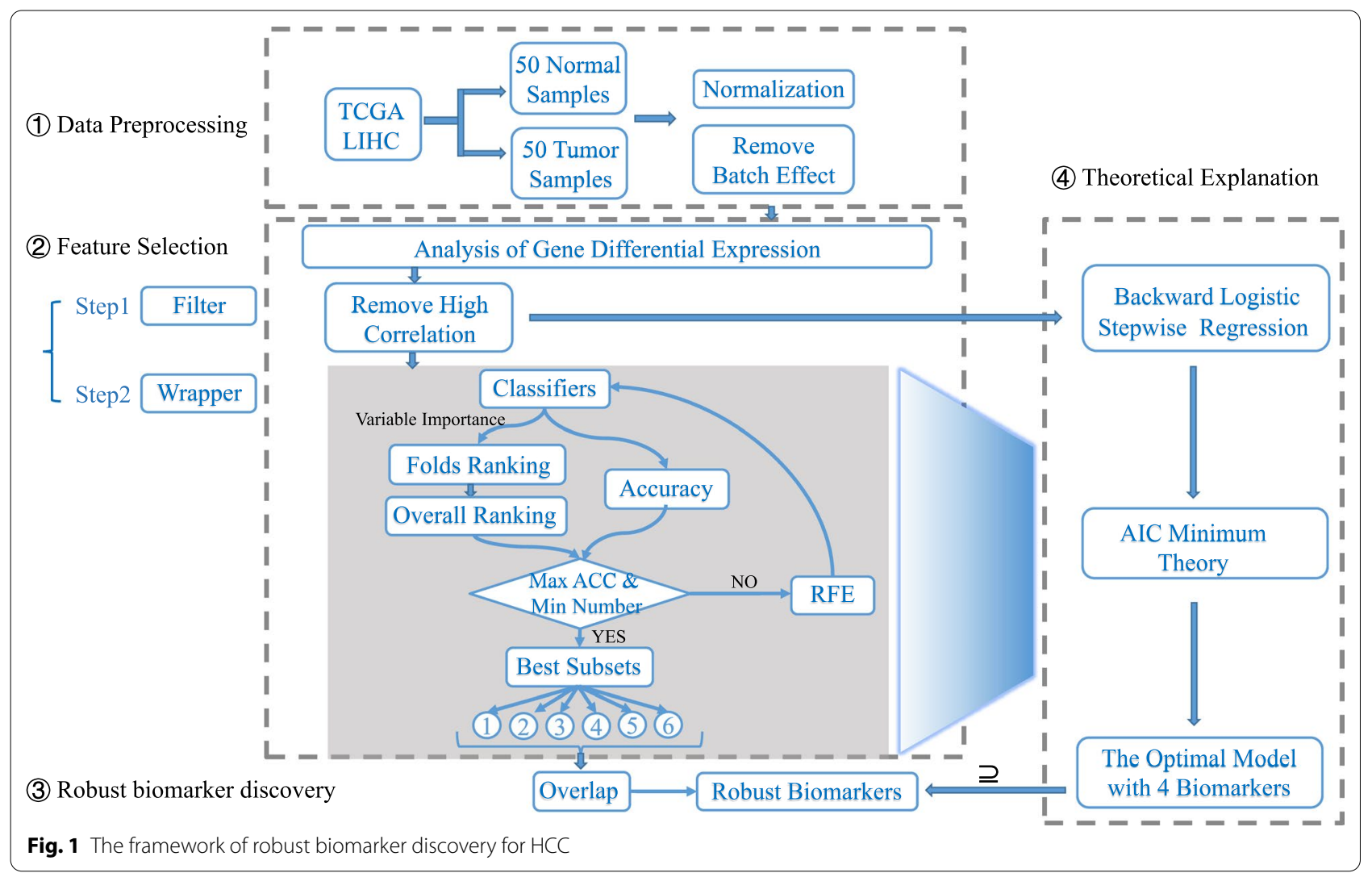

as a covariate. The data and code used in this paper are available at: http://www.github.com/zpliulab/RobMarker.

\section{Feature selection}

Filter, wrapper and embedded techniques are three major types of feature selection methods [17]. In this work, the process of selecting biomarkers mainly consists of two substeps. The first is a filter and the second is a wrapper. In the filtering, we firstly select the genes with differential expression. After data normalization, the differentially expressed genes which meet the threshold requirements form the candidate pool of biomarkers [15]. We set the threshold condition as false discovery rate $(\mathrm{FDR})<0.01$ (1\%), $P$ value $<0.01$ and Fold Change $>3$. Secondly, we remove one of the redundant features that have a high correlation value with each other. For two genes, if the Pearson's correlation coefficient between them is greater than 0.65 , the gene with the higher mean absolute value is deleted [9].

Wrapper-based feature selection is the focus of our approach. Wrapper adopts the recursive feature selection method based on cross-validation to further select biomarkers from the gene pool which is obtained in the previous step. Here, 6 classical classification algorithms are tested, i.e., Adaboost, KNN, NB, NN, RF, SVM, which are combined with feature selection respectively. Each method is recorded to select the feature subset with the best classification accuracy and the least number of features [18].

\section{Recursive feature elimination based on cross-validation (RFE-CV)}

It has been found that biomarkers selected by RFE$\mathrm{CV}$ have better classification performance than those selected by RFE [19]. For details, the process of RFE$\mathrm{CV}$ can be divided into two procedures [20]. In the first, we apply tenfold cross-validation to get the feature importance ranking. For each feature, we summarize its importance score in every folded dataset to obtain an overall ranking. In the second, the features at the last places in the overall ranking are gradually deleted, until all the features are removed. The best classification subset is determined at the end of the algorithm. In this study, we use the RFE with tenfold cross-validation, so that each feature gets 10 importance scores. We take an average of them for a consensus ranking. Each time a feature is deleted, the classification accuracy of the model is calculated accordingly. The subset with the highest classification accuracy and the least number of features is regarded as the best feature subset. For each 
feature, we record its importance in each fold to track the change of importance over different folds. Thus, RFE-CV provides more probabilistic estimates of the importance of predictive variables than ranking based only on a single dataset [18].

We find the measure of feature importance by classifiers can directly affect the ranking of features. NN calculates the feature importance based on the connection weights between neurons in hidden layers. It divides the hidden-output connection weight of each hidden neuron into components related to each input neuron. The importance of its characteristics is based on a product that is the absolute value of the hidden-output layer connection weight multiplied by the absolute value of the hidden-input layer connection weight. For the importance of a feature, the sum of its proportion in each neuron is calculated based on the product. Then we calculate the ratio of all the features to the sum of their weights in each neuron [21]. In general, $\mathrm{NN}$ determines the relative importance of a feature by identifying all weighted connections between the nodes of interest [21]. We set the number of hidden neurons of NN classifier as 8 and the maximum number of feedback iterations as 30 [22]. In $\mathrm{RF}$, we set the number of decision trees to be 500 [23]. The calculation of feature importance is based on the average value of the difference between the two out-ofpocket error rates of each decision tree [20]. After adding noise to a particular feature, the features that make the accuracy more affected, and often more significant. The argument is that if a feature is important, then the change will greatly affect the test error. If the test error does not change much, then the feature is not important. Some methods calculate the importance of features according to their contributions to the classification performance. For instance, Adaboost, KNN, NB and SVM rank the importance of features according to the AUC values contributed by each feature. Although these four classifiers rank the features according to the classification accuracy obtained by training the model with single feature, the models established by different classifiers for the same feature are different because of different calculation rules. We set the number of weak decision tree classifiers in Adaboost to 10 [24], the parameter $\mathrm{k}$ in $\mathrm{KNN}$ to 3 [25], and the kernel function in SVM to be linear [26]. NB classifier has no predetermined parameters [27].

\section{Robust biomarker discovery}

For the selection of biomarkers, the repeatability of biomarkers is as important as the classification accuracy of the constructed models. The feature selection method based on wrapper is a strategy guided by machine learning algorithm [28]. Machine learning algorithm is a black box, and we don't know how it calculates the importance of features. When a feature is calculated using different importance calculations and shows good importance in different ways, we acknowledge that it is a feature that makes sense in the model. If it is only important in a particular method, then we think its importance is not universal. It is more likely to be only related to the computational process of the method and not to the nature of the model. The aim here is to establish a method for robust biomarker discovery. To achieve the reproducibility of biomarkers, we regard the genes with two or more occurrences in the 6 selected-feature subsets as identified biomarkers. We calculate the number of intersection features between the two subsets and perform a hypergeometric test to calculate the significance $P$ value of overlapping. Then we analyze the biomarkers that appear in different subsets several times to verify the effectiveness of our method. The more times a feature is selected in different ways, the better its repeatability. We train the model with genes that appear more than four times and then make predictions on independent datasets.

\section{Backward logistic stepwise regression with AIC}

Typically, the feature selection procedure of RFE is a black box. To shed light on the black box, we propose a theoretical explanation of the selection process. We employ AIC value to explain the feature selection process in machine learning and use a backward logistic stepwise regression to explain the results of feature selection.

Stepwise regression is one of the main methods for model selection which has relatively sufficient theoretical basis [29]. By recording the process of feature selection, each step of RFE-CV process is measured by AIC. In this study, the stepwise regression via AIC minimum theory is applied to the candidate pool to select the model with the minimum AIC value.

In order to introduce the maximum likelihood method into the multi-model selection problem, Akaike proposed AIC which is suitable for a wide range of problem. It makes us use the backward stepwise regression method combined with AIC [29]. The problem we interested is a binary classification, so we choose logistic regression for an easy explanation. Logistic regression uses the method of maximizing likelihood estimation and gradient descent method to solve parameters to achieve the purpose of data dichotomy. The goal is to find the best fitting model to describe the relationship between dependent variables and a set of independent (predicted or interpreted) variables [30].

Firstly, we put all features in the candidate set into the model. Secondly, logistic regression is performed on these features [31]. The algorithm tries to remove one of the independent variables from the model to see if there is a significant change in the AIC values. And then it 
removes the variable that minimizes AIC. This process is repeated until no arguments meet the elimination criteria. Our goal is to find a model with the smallest AIC value.

AIC is widely used in the model selection in statistics [14]. When the goodness of fit of the model is the same degree, the model with fewer variables is preferred [14]. The parsimony principle of model selection is similar to that of RFE-CV. For completeness, we make a brief introduction of the calculation process of AIC. More detailed calculation steps refer to the supplementary material.

Suppose that a random variable $Y$ has a probability density function $f(y \mid \theta)$, and $\theta$ is the parameter vector. The likelihood function of $\theta$ is defined as $L(\theta)=f\left(y_{1} \mid \theta\right) f\left(y_{2} \mid \theta\right) \ldots f\left(y_{N} \mid \theta\right)$. The $g(y)$ is the probability density function that describes the true distribution of $Y$. Here $\hat{\theta}$ is considered as the estimate of $\theta$ that maximizes the logarithmic likelihood function $l(\theta)=\ln L(\theta)$. Because of $l(\theta)=\sum \ln f\left(y_{i} \mid \theta\right)$, then we can get

$$
\frac{1}{N} l(\theta) \rightarrow E \ln f(Y \mid \theta)=\int g(y) \ln f(y \mid \theta), \quad N \rightarrow \infty .
$$

We introduce $m=\frac{\max L\left(\theta_{0}\right)}{\max L(\hat{\theta})}$ by means of the methods in the literature [14]. Then we get

$$
-2 \ln m=-2 \ln \frac{\max L\left(\theta_{0}\right)}{\max L(\hat{\theta})}=\Sigma\left[\ln \frac{f(y \mid \hat{\theta})}{f\left(y \mid \theta_{0}\right)}\right]^{2} .
$$

When $N \rightarrow \infty,-2 \ln m$ asymptotically obeys the chisquare distribution of $\mathrm{t}$ degrees of freedom. The $t$ is the dimension of the parameter vector $\theta$. In other words, it is $\left.E\left\{2\left[l(\hat{\theta})-l\left(\theta_{0}\right)\right]\right)\right\}=t$. The formulas are as follows

$$
\begin{aligned}
2 l(\hat{\theta}) & =2 \Sigma \ln f\left(y_{i} \mid \hat{\theta}\right)=2 N \int f\left(y_{i} \mid \hat{\theta}\right) \ln f\left(y_{i} \mid \hat{\theta}\right) d y \\
2 E^{*} l\left(\theta_{0}\right) & =2 \Sigma \int f\left(x_{i} \mid \theta_{0}\right) \ln f\left(x_{i} \mid \theta_{0}\right) d x \\
& =2 N \int f\left(x_{i} \mid \theta_{0}\right) \ln f\left(x_{i} \mid \theta_{0}\right) d x .
\end{aligned}
$$

From Formula (3), we know that the adjacent shape of $2 l(\theta)$ at $\theta=\theta$ can be approximated by the adjacent shape of $2 E^{*} l(\theta)$ at $\theta=\theta_{0} .2 l(\theta)$ and $2 E^{*} l(\theta)$ are approximated by quadric surfaces with vertices $\hat{\theta}_{\hat{A}}$ and $\theta_{0}$. That means that $2 E^{*} l\left(\theta_{0}\right)$ is $t$ higher than $2 E^{*} l(\theta)$ on average. So the estimate of $E\left\{2 E^{*} l(\hat{\theta})\right\}=2 N E^{*} E \ln f(Y \mid \hat{\theta})$ is $2 l(\hat{\theta})-2 t$. Then we can get

$$
A I C=-2 l(\hat{\theta})+2 t .
$$

When there is a big difference between the two models, the first term plays a major role in the difference. When the models are not very different, the second term plays a major role. A more detailed derivation can be found in the Additional file 1.

\section{Results}

\section{Classification and feature selection}

We obtain the 6 best classification subsets corresponding to the 6 machine learning algorithms. The feature subsets contain the least number of features but can enable the machine learning algorithms to achieve their best classification performances individually. When each classifier reaches its maximum classification accuracy, we obtain its classification performance. Five evaluation parameters, sensitivity (SN), specificity (SP), F1-score, accuracy (ACC) and AUC (area under curve) are used. The ROC curves are shown in Fig. 2. The corresponding evaluation metrics are shown in Table 1. From them, we find that each classifier achieves good classification performance. The classification accuracy of $\mathrm{NB}$ and $\mathrm{RF}$ reaches 0.99 , i.e., only one sample is misclassified. It demonstrates the effectiveness of our method in selecting biomarkers for classifying HCC and control samples.

Because of the different ways of calculating feature importance, the $6 \mathrm{RFE}-\mathrm{CV}$ methods select the best subset individually. Thus, the selected feature subsets contain different genes. In the feature selection process, we will iteratively obtain a new model after removing a feature. In order to make full use of all samples,

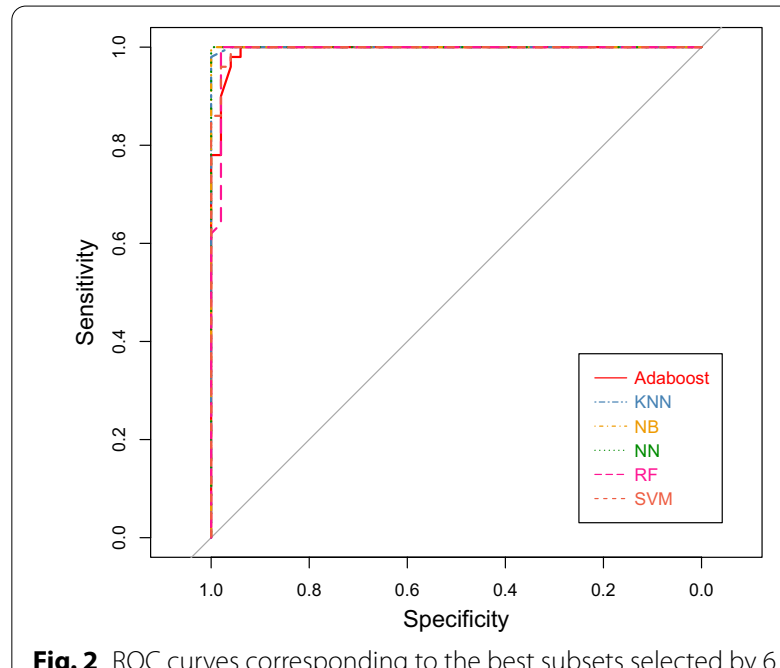

Fig. 2 ROC curves corresponding to the best subsets selected by 6 classification algorithms 
Table 1 The classification performance of the 6 classifiers

\begin{tabular}{|c|c|c|c|c|c|c|}
\hline Method & \# of gene & SN & SP & F1-score & ACC & AUC \\
\hline Adaboost & 21 & 0.940 & 1.00 & 0.969 & 0.970 & 0.994 \\
\hline KNN & 62 & 0.960 & 1.00 & 0.979 & 0.980 & 0.999 \\
\hline NB & 12 & 0.980 & 1.00 & 0.989 & 0.990 & 1.00 \\
\hline NN & 63 & 0.960 & 1.00 & 0.979 & 0.980 & 1.00 \\
\hline RF & 13 & 0.980 & 1.00 & 0.989 & 0.990 & 0.993 \\
\hline SVM & 57 & 0.960 & 1.00 & 0.979 & 0.980 & 0.996 \\
\hline
\end{tabular}
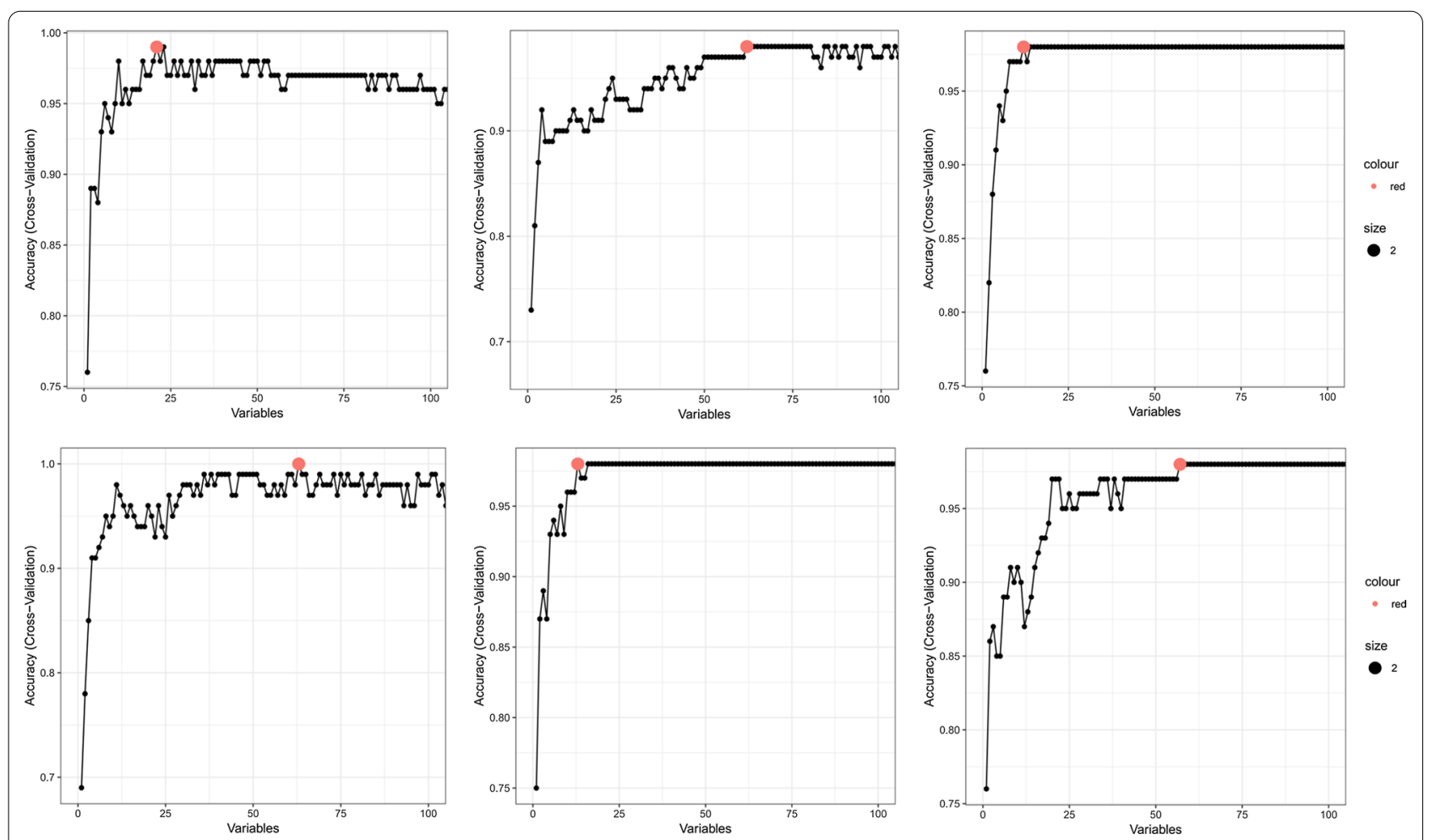

Fig. 3 The process of RFE-CV in 6 classifiers. Red point refers to these features with the maximum accuracy

we implement tenfold cross-validation classification for the new model. By changing the number of features for a model, different classification accuracy can be obtained. Figure 3 illustrates the correspondence between classification accuracy and feature number. The red points annotate the best feature subset with the highest classification accuracy and the least number of genes. Among them, the subset selected by NB contains the least number of features, namely 12 genes, followed by 13 genes selected by RF classifier.

\section{Discovery of biomarkers}

We compare 6 RFE-CV methods and find a lot of overlaps between the optimal gene subsets. The specific relationship between them is shown in Fig. 4. As described, genes in two or more selected subsets are regarded as the identified biomarkers. A total of 110 genes are selected by the former 6 methods, and 60 genes are contained in the overlaps, which are considered as biomarkers. Among them, 32 genes are contained in 3 or more optimal feature subsets, which are considered to have more functional significance. 


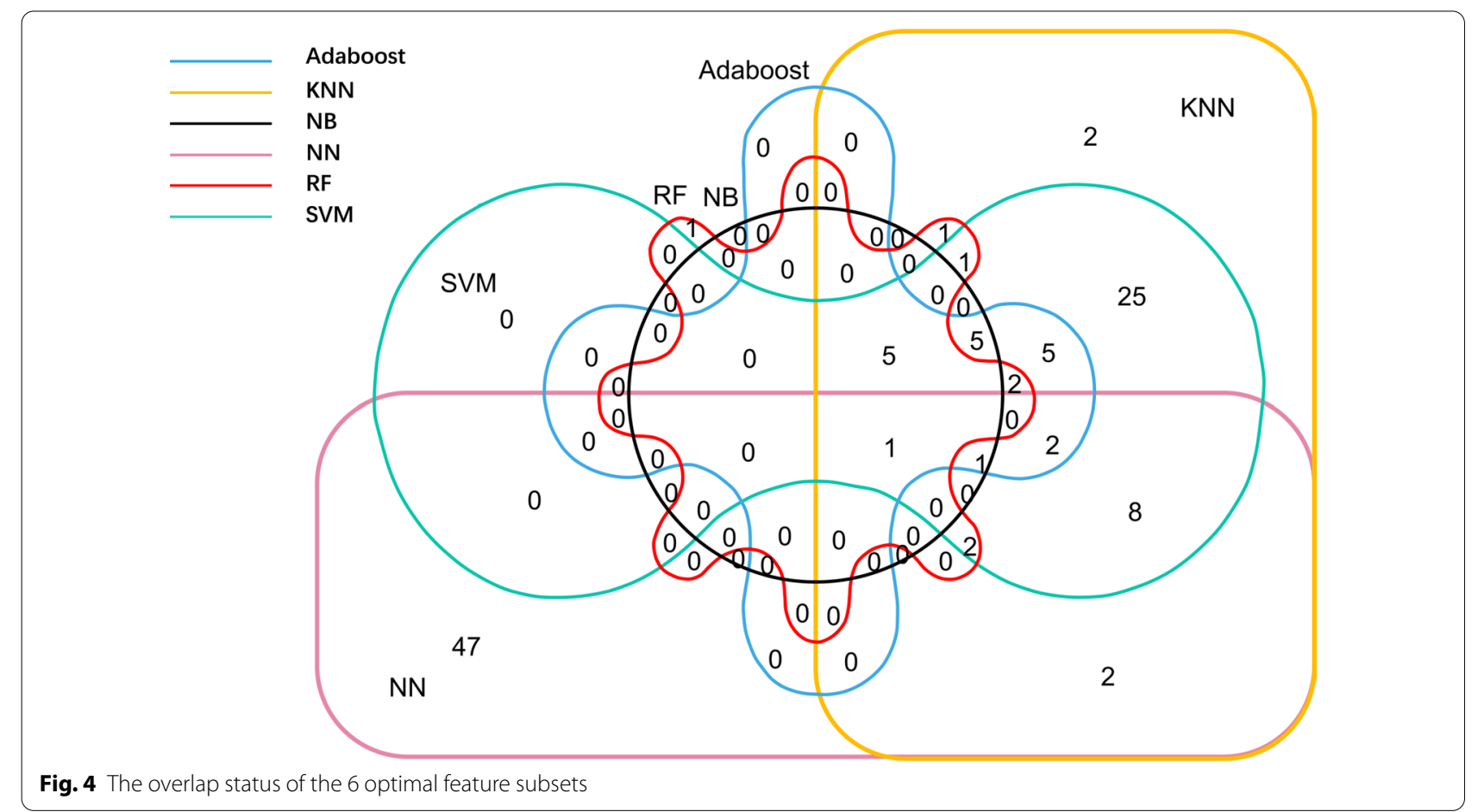

Firstly, the 6 optimal subsets have a common biomarker, SKAP1. Also, SKAP1 is the last feature of the stepwise regression deletion, although it is not included in the final four biomarkers identified by stepwise regression. Studies have revealed that SKAP1 is a gene involved in the biological process of immune T cells [32]. It is also shown to be related to the signaling pathways of HCC [33].

Secondly, we find that the four optimal feature subsets selected by Adaboost, KNN, NB and SVM classifiers have inclusion relations. Among them, subsets with a larger number of features contain the subsets with a smaller number of features. The reason is underlying their same feature ranking method. The four classifiers measure the importance of each feature based on the AUC value when each feature is classified separately. By comparing the genes in the four identified optimal feature subsets, we find most of these features rank at the same places in the four classifiers. The important genes identified by different classifiers indicate the effectiveness and consistency of different feature selection strategies.

Moreover, we find that there are a lot of overlaps between the features selected by RF and the features selected by the four classifiers. 6 out of 12 features selected by NB appear in the 13 features selected by RF. The feature subsets selected by $\mathrm{NN}$ and $\mathrm{KNN}$ have the largest intersection, with 16 features. The overlap between two subsets is shown in Table 2. We employ the hypergeometric distribution test for achieving its statistical significance $P$ value [34].

Table 2 The number of overlapping features and the corresponding significance $P$ values

\begin{tabular}{|c|c|c|c|c|c|c|c|}
\hline Overlap & Adaboost & KNN & NB & NN & $\mathrm{RF}$ & SVM & SR \\
\hline Adaboost & 21 & 21 & 12 & 4 & 8 & 21 & 2 \\
\hline KNN & $<1 \mathrm{e}-6$ & 62 & 12 & 16 & 12 & 57 & 4 \\
\hline NB & $<1 \mathrm{e}-6$ & $<1 \mathrm{e}-6$ & 12 & 2 & 6 & 12 & 2 \\
\hline NN & $6 e-2$ & $<1 \mathrm{e}-6$ & $2.1 \mathrm{e}-1$ & 63 & 3 & 14 & 3 \\
\hline RF & $<1 \mathrm{e}-6$ & $<1 \mathrm{e}-6$ & $<1 \mathrm{e}-6$ & $6 e-2$ & 13 & 11 & 0 \\
\hline SVM & $<1 e-6$ & $<1 \mathrm{e}-6$ & $<1 \mathrm{e}-6$ & $1.43 e-5$ & $<1 \mathrm{e}-6$ & 57 & 4 \\
\hline SR & $5.36 \mathrm{e}-4$ & $1.82 \mathrm{e}-5$ & $9.95 e-4$ & $1.3 e-3$ & 1 & $1.55 e-5$ & 4 \\
\hline
\end{tabular}


Table 3 The last 5 genes deleted by stepwise regression

\begin{tabular}{lllll}
\hline Step & Deviance & Resid. Dev & P value & AIC \\
\hline ID2B & $7.72 \mathrm{e}-11$ & $1.91 \mathrm{e}-09$ & $1.73 \mathrm{e}-17$ & $18+1.91 \mathrm{e}-09$ \\
PMP2 & $4.55 \mathrm{e}-10$ & $2.37 \mathrm{e}-10$ & $1.05 \mathrm{e}-19$ & $16+2.37 \mathrm{e}-10$ \\
MUC6 & $5.45 \mathrm{e}-10$ & $2.91 \mathrm{e}-10$ & $1.59 \mathrm{e}-07$ & $14+2.91 \mathrm{e}-10$ \\
C1QL1 & $1.03 \mathrm{e}-09$ & $3.94 \mathrm{e}-09$ & $1.74 \mathrm{e}-24$ & $12+3.94 \mathrm{e}-09$ \\
SKAP1 & $1.25 \mathrm{e}-08$ & $1.64 \mathrm{e}-08$ & $4.19 \mathrm{e}-13$ & $10+1.64 \mathrm{e}-08$ \\
\hline
\end{tabular}

Some biomarkers that appear more frequently in the selected subsets are considered as important feature genes, which should be explored their biological functional significance in later sections.

\section{Statistical interpretation of feature selection in machine learning}

In the candidate biomarker pool, we perform a backward logistic stepwise regression on 886 differentially expressed genes [35]. The AIC values are used to measure the quality of the model iteratively. After putting all the features in the model, we remove sequentially the ones that reduce the AIC value with the fastest speed. At the end of the algorithm, the final best model has the smallest AIC value. It selects four feature genes, namely COL9A1, PHOSPHO1, EGFL6 and OXT. All the four features are included in the biomarker set selected by the former 6 REF-CV methods. Since the stepwise regression selects very few features, we analyze it from another perspective. We find that the relatively important features deleted near the end of stepwise regression are actually included in the selected biomarker set. Moreover, the $P$ values of these features are very small. It indidates they are significantly differentially expressed genes. From the beginning of stepwise regression, there is a tendency for the latter deletion feature to have a smaller $P$ value. Table 3 lists the lastdeleted five features in the stepwise regression process, and we can determine that they are indeed included in our biomarker collection.

We also analyze the overlap between the four features selected by the backward logistic stepwise regression and the optimal subsets of each classifier. The four features of the backward logistic stepwise regression are fully contained in the feature subsets of KNN and SVM, and three features are in the subset of NN. The overlap of backward logistic stepwise regression and the other optimal subsets are illustrated in Tables 2 and 3 respectively.

Synchronization with the iterative elimination of RFE$\mathrm{CV}$, for each deletion of a feature in the iteration, logistic regression is performed on the new model and its AIC value is calculated correspondingly. By calculating AIC

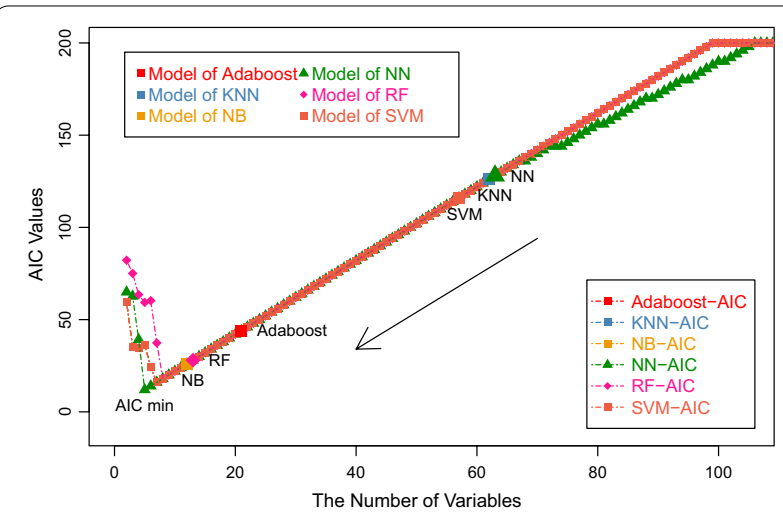

Fig. 5 The trend of AIC value in the feature selection process

values, we statistically interprete the feature selection process for each classifier. The relationship between AIC values and feature numbers is shown in Fig. 5.

As illustrated in Fig. 5, AIC values gradually decrease with the decreasing number of features. Except the NN classifier, AIC values based on the other five classifiers all decline at a slope of 2 . When the number of features is large, the features fit the model roughly the same. The number of features has a large impact on the AIC value. When the AIC value drops to a certain point $(<20)$, it tends to increase sharply. The turning point has the minimum AIC value. When the number of features in the subset is less than the turning point, the goodness of fit to the model will deteriorate. In this case, the number of features will not be the dominant factor, thus the AIC value will increase.

In Fig. 5, we also annotate the AIC values of models when each classifier identifies its optimal feature subset. NB has the lowest AIC value, followed by RF. Although the classification performances obtained by the models trained by KNN, NN and SVM are good, their corresponding minimum AIC values are relatively large due to the large number of features. Using simple machine learning methods to make feature selection, biomarkers cannot be excellently selected because redundant features may still exist. Therefore, AIC can be combined with the RFE method as a mentor, guiding the feature selection process for robust biomarker discovery.

According to the change trend of AIC value shown in Fig. 5, we theoretically explain the feature selection process. As is known to all, the feature selection process based on AIC is a process in which the goodness of model fit increases and the number of features decreases [14]. Through our method, we prove that the feature selection process of biomarker discovery based on machine learning is also such a process. 
Table 4 Some genes and their dysfunctions from the interactions of selected feature subsets of different methods

\begin{tabular}{|c|c|c|}
\hline Gene & Subset & Function \\
\hline SKAP1 & 6 methods & SKAP1 encodes a T cell adaptor protein and it is involved in HCC signaling pathways $[32,33]$ \\
\hline EPHB1 & SVM, KNN, NN & $\begin{array}{l}\text { Ephrin-B1 participates in the tumor progression through promoting the formation of new vessels of } \\
\text { HCC [36] }\end{array}$ \\
\hline STC2 & NN, SVM, KNN & STC2 is overexpressed in HCC and acts as a potential oncoprotein [37] \\
\hline CDHR2 & NN, SVM, KNN & $\begin{array}{l}\text { CDHR2 is highly expressed in HCC para-carcinoma tissue, but is weakly expressed in tumors. It is } \\
\text { found to inhibit tumor growth [38] }\end{array}$ \\
\hline FAM134B & NN, SVM, KNN & FAM134B works as a tumor inhibitor and inhibits cancer growth in vitro and in vivo [39] \\
\hline MUC6 & RF, NN, SVM, KNN & MUC6 encodes a member of the mucin protein family. It is a biomarker gene of many cancers [40] \\
\hline PHOSPHO1 & $\begin{array}{l}\text { Adaboost, NB, NN, SVM, KNN, Step- } \\
\text { wise Regression }\end{array}$ & PHOSPHO1 is associated with hepatitis B [41] \\
\hline OXT & NN, SVM, KNN, Stepwise Regression & $\begin{array}{l}\text { OXT is found to regulate cell proliferation. It is a key differential gene in nonalcoholic fatty liver } \\
\text { disease [42] }\end{array}$ \\
\hline
\end{tabular}

\section{Validation of biomarkers}

We verify the effectiveness of the identified biomarkers in three ways, which will also prove the effectiveness of our proposed method. The genes in the intersection are identified as the robust biomarkers. The higher the frequency of gene emergence, the more likely it is to precisely diagnose the disease.

Firstly, we make a statistics of the biological functions of some important genes. Some genes have been verified to be closely associated with the occurrence and development of HCC. Table 4 lists some representative biomarker genes in the overlaps. From the dysfunctions of genes listed in Table 4, we can conclude that the method of identifying biomarkers from overlaps is effective.

Secondly, we validate the features selected by the bestperforming classifiers on independent data sets. Based on the former results, we find that NB-RFE-CV achieves the best classification performance. Specifically, it selects the least number of feature subsets and obtain a better AUC value than the other five classifiers. Moreover, the optimal subset corresponding to NB for logistic regression has the minimum AIC value. To verify the results are not caused by overfitting, we use 12 genes selected by NB to classify samples of an independent dataset. We download another HCC dataset from GEO in NCBI with ID GSE25097 [9]. We use the former 100 TCGA samples as the training set and GSE25097 as the testing data set. Figure 6a shows the classification performance of the 12 features. The metrics of SN, SP, F1-score, ACC and AUC are also shown. The results of cross-dataset validation demonstrate that the former good classification ability is not caused by overfitting, and that our proposed method of identifying biomarkers is efficient.

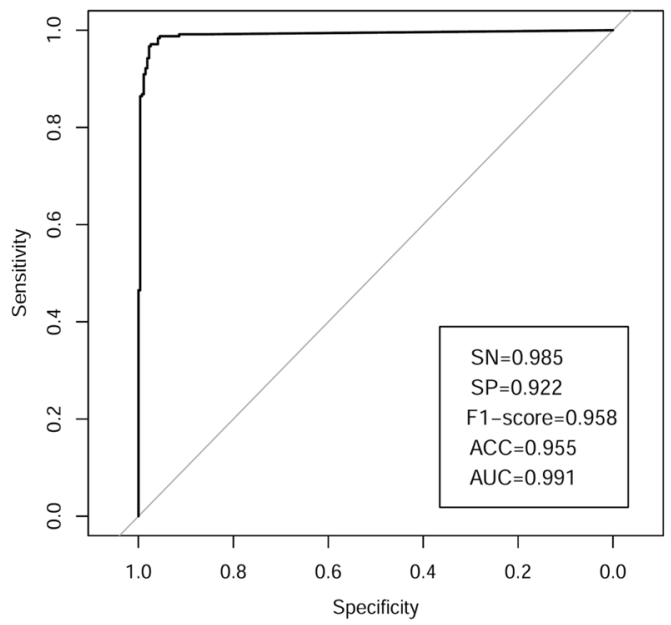

a Classification results of 12 features selected by NB.

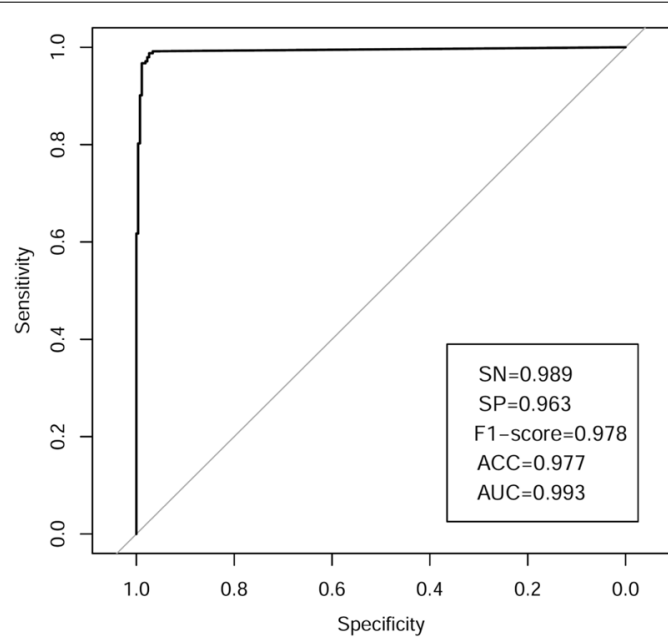

b Classification results of 17 features in more than 4 overlaps.

Fig. 6 The ROC curves of the trained NB classifier in the independent data GSE25097 
What's more, it is also necessary to check the model trained by overlap features on independent datasets. There are 17 features that appear more than 4 times in the 6 feature subsets. We use these 17 features to train on the balanced data set of TCGA and test on the independent data set GSE25097. In the same way, NB classifier is selected. Figure $6 \mathrm{~b}$ shows the classification perforamcne of 17 features.

We compare the two models and find that 17 features in overlap could achieve better classification performance. The latter's five evaluation indicators are all higher than the former ones. Our proposed method greatly improves the elimination of false positive feature genes. To sum up, the three verifications further justify the effectiveness of our proposed method.

\section{Discussion}

In this paper, we proposed a robust biomarker discovery framework via multiple feature selection methods. The set of differentially expressed genes provides a pool of biomarker candidates. We applied the RFE-CV feature selection methods based on 6 different classification algorithms to select diagnostic biomarkers from the candidate pool. The 6 classifiers respectively get the importance ranking of features and further obtain the best feature subsets. Theoreticallly, AIC was employed to explain the feature selection process of machine learning. In the process of feature reduction, AIC value also decreases, indicating that our feature selection process is statistically interpretable. For the 6 optimal feature subsets selected, we found out their overlaps that serve as robust biomarkers. These discovered biomarkers have been shown to be closely related to the occurrence and development of HCC.

To achieve a statistical explanation of machine learning and feature selection, the genes in the candidate pool are simultaneously regressed step by step according to the AIC minimum theory. We found the subset of genes selected in the adapative regression are completely included in the subset of genes selected by feature selection in machine learning. The consistency further indicates the important features play an important role in training different methods. According to the trend of AIC value changing in the feature selection process, it can be found that the selected features may not be the most concise one only by machine learning. For example, the optimal subsets selected by $\mathrm{NN}, \mathrm{KNN}$ and SVM contain relatively larger number of biomarker genes. In the model fitting of these selected subsets, the relationship between the AIC goodness and the number of features is not optimal. It is clear that some redundant feature genes still exist in these subsets. Ideally, the model with a minimum AIC value should be selected to ensure the best classification. Although our method can explain the feature selection process based on machine learning, the exploration of explicable feature selection process should go further. We will also continue to study the interpretability of feature selection.

Moreover, we found feature genes contained in more subsets make more biological sense. The quality of feature selection is improved by the intersection of biomarkers. Through the previous experiments, we can prove that our method greatly improves the classification performance, especially the problem of high false positives that the current classification model often achieves.

\section{Conclusion}

In summary, we presented a method for robust biomarker discovery from RNA-seq data based on feature selection with statistical validation. Not only do we provide a statistical interpretation of the machine-learningbased feature selection process, but also the results of gene function enrichment analysis and the validation of independent data sets provide a compelling argument for our approach. More importantly, we proposed a solution to the problem of feature selection instability. Our method is not only applicable to the discovery of HCC biomarkers, but also to the discovery of other disease biomarkers.

\section{Abbreviations}

HCC: Hepatocellular carcinoma; TCGA: The cancer genome atlas; RFE: Recursive feature elimination; RFE-CV: Recursive feature elimination cross-validation; AIC: Akaike information criterion; KNN: K-nearest neighbor; NB: Naïve Bayes; NN: Neural network; RF: Random forest; SVM: Support vector machine; LIHC: Liver Hepatocellular Carcinoma; RNA-seq: RNA sequencing; GDC: Genomic Data Commons; FDR: False discovery rate; SN: Sensitivity; SP: Specificity; ACC: Accuracy; AUC: Area under curve.

\section{Supplementary Information}

The online version contains supplementary material available at https://doi. org/10.1186/s12920-021-00957-4.

Additional file 1. Supplementary material for the calculation process of AIC

\section{Acknowledgements}

Thanks are due to the anonymous reviews for their insightful comments which have greatly improved our manuscript.

\section{About this supplement}

This article has been published as part of BMC Medical Genomics Volume 14 Supplement 1, 2021: Proceedings of the 2019 International Conference on Intelligent Computing (ICIC 2019): medical genomics. The full contents of the supplement are available online at https://bmcmedgenomics.biomedcentral. com/articles/supplements/volume-14-supplement-1. 


\section{Authors' contributions}

$\mathrm{ZZ}$ performed the experiments, analyzed the data and wrote the paper. ZL conceived and designed the experiments, and wrote the paper. Both authors read and approved the final manuscript.

\section{Funding}

National Natural Science Foundation of China (NSFC) under Grant Numbers 61973190 and 61572287; National Key Research and Development Program of China under Grant Number 2020YFA0712402; Shandong Provincial Key Research and Development Program (Major Scientific and Technological Innovation Project, 2019JZZY010423); Natural Science Foundation of Shandong Province of China under Grant Number ZR2020ZD25; the Innovation Method Fund of China (Ministry of Science and Technology of China) under Grant Number 2018IM020200; the Program of Qilu Young Scholars of Shandong University. Publication costs are funded by NSFC. The funding bodies had no role in the design of the study, collection, the interpretation of data and in writing the manuscript.

\section{Availability of data and materials}

The Cancer Genome Atlas (TCGA) datasets for Liver Hepatocellular Carcinoma [Project ID: TCGA-LIHC, Project Name: Liver Hepatocellular Carcinoma (dbGaP Study Accession: phs000178)] supporting the conclusions of this article are available in the repository in Genomic Data Commons Data Portal at https:// portal.gdc.cancer.gov/. The validation data set GSE25097 is available at https:// www.ncbi.nlm.nih.gov/. The code used in this paper is available at http:// www.github.com/zpliulab/RobMarker.

\section{Declarations}

Ethics approval and consent to participate

Not applicable.

\section{Consent for publication}

Not applicable.

\section{Competing interests}

The authors have no competing interests to declare.

\section{Author details}

${ }^{1}$ Department of Biomedical Engineering, School of Control Science and Engineering, Shandong University, Jinan 250061, Shandong, China. ${ }^{2}$ Center for Intelligent Medicine, Shandong University, Jinan 250061, Shandong, China.

Received: 29 October 2020 Accepted: 8 April 2021

Published online: 25 August 2021

\section{References}

1. Cancer Genome Atlas Research Network. Comprehensive and integrative genomic characterization of hepatocellular carcinoma. Cell. 2017;169(7):1327-1341.e1323.

2. Tong Z, Zhou Y, Wang J. Identifying potential drug targets in hepatocellular carcinoma based on network analysis and one-class support vector machine. Sci Rep. 2019;9(1):10442.

3. Balogh J, Victor D 3rd, Asham EH, Burroughs SG, Boktour M, Saharia A, Li X, Ghobrial RM, Monsour HP Jr. Hepatocellular carcinoma: a review. J Hepatocell Carcinoma. 2016;3:41-53.

4. Tomczak K, Czerwińska P, Wiznerowicz M. The Cancer Genome Atlas (TCGA): an immeasurable source of knowledge. Contemp Oncol (Pozn). 2015;19(1A):A68-77.

5. Liu Z-P. Identifying network-based biomarkers of complex diseases from high-throughput data. Biomark Med. 2016;10(6):633-50.

6. Libbrecht MW, Noble WS. Machine learning applications in genetics and genomics. Nat Rev Genet. 2015;16:321.

7. Abeel T, Helleputte T, Van de Peer Y, Dupont P, Saeys Y. Robust biomarker identification for cancer diagnosis with ensemble feature selection methods. Bioinformatics. 2009:26(3):392-8.
8. Książek W, Abdar M, Acharya UR, Pławiak P. A novel machine learning approach for early detection of hepatocellular carcinoma patients. Cogn Syst Res. 2019;54:116-27.

9. Zhang Z, Liu Z-P. Identifying cancer biomarkers from high-throughput RNA sequencing data by machine learning. In: International conference on intelligent computing: 2019. Springer; 2019: 517-28.

10. Ruffalo M, Thomas RA, Chen J, Lee AV, Oesterreich S, Barjoseph Z. Network-guided prediction of aromatase inhibitor response in breast cancer. PLoS Comput Biol. 2019;15(2):e1006730.

11. Fan S, Tang J, Li N, Zhao Y, Ai R, Zhang K, Wang M, Du W, Wang W. Integrative analysis with expanded DNA methylation data reveals common key regulators and pathways in cancers. npj Genom Med. 2019;4(1):2.

12. Fan S, Tang J, Tian Q, Wu C. A robust fuzzy rule based integrative feature selection strategy for gene expression data in TCGA. BMC Med Genom. 2019;12(1):25-33.

13. Tansey W, Veitch $V$, Zhang H, Rabadan R, Blei DM. The holdout randomization test: principled and easy black box feature selection. arXiv preprint arXiv:181100645. 2018.

14. Akaike H. Factor analysis and AIC. In: Selected papers of Hirotugu Akaike. Springer; 1987. p.371-86.

15. Wenric S, Shemirani R. Using supervised learning methods for gene selection in RNA-seq case-control studies. Front Genet. 2018;9:297.

16. Anders $S, H u b e r W$. Differential expression analysis for sequence count data. Genome Biol. 2010;11(10):R106.

17. Li J, Cheng K, Wang S, Morstatter F, Trevino RP, Tang J, Liu H. Feature selection: a data perspective. ACM Comput Surv. 2017;50(6):1-45.

18. Kuhn M. Building predictive models in $\mathrm{R}$ using the caret package. J Stat Softw. 2008;28(5):1-26

19. Zhang F, Kaufman HL, Deng Y, Drabier R. Recursive SVM biomarker selection for early detection of breast cancer in peripheral blood. BMC Med Genom. 2013;6(1):S4

20. Genuer R, Poggi J-M, Tuleau-Malot C. Variable selection using random forests. Pattern Recogn Lett. 2010;31(14):2225-36.

21. Gevrey M, Dimopoulos I, Lek S. Review and comparison of methods to study the contribution of variables in artificial neural network models. Ecol Model. 2003;160(3):249-64.

22. Hecht-Nielsen R. Theory of the backpropagation neural network. In: Neural networks for perception. Elsevier; 1992. 65-93.

23. Breiman L. Random forests. Mach Learn. 2001;45(1):5-32.

24. Freund $Y$, Schapire RE. Experiments with a new boosting algorithm. In: ICML: 1996: Citeseer; 1996. p. 148-156.

25. Cover T, Hart P. Nearest neighbor pattern classification. IEEE Trans Inf Theory. 1967;13(1):21-7.

26. Cortes C, Vapnik V. Support-vector networks. Mach Learn. 1995;20(3):273-97.

27. Domingos P, Pazzani M. On the optimality of the simple bayesian classifier under zero-one loss. Mach Learn. 1997;29(2):103-30.

28. Gangavarapu T, Patil N. A novel filter-wrapper hybrid greedy ensemble approach optimized using the genetic algorithm to reduce the dimensionality of high-dimensional biomedical datasets. Appl Soft Comput. 2019:81:105538.

29. Yamashita T, Yamashita K, Kamimura R. A stepwise aic method for variable selection in linear regression. Commun Stat Theory Methods. 2007;36(13):2395-403

30. Hosmer DW Jr, Lemeshow S, Sturdivant RX. Applied logistic regression, vol. 398. New York: Wiley; 2013.

31. Steyerberg EW, Eijkemans MJC, Habbema JDF. Stepwise selection in small data sets: a simulation study of bias in logistic regression analysis. J Clin Epidemiol. 1999;52(10):935-42.

32. Raab M, Wang H, Lu Y, Smith X, Wu Z, Strebhardt K, Ladbury JE, Rudd CE. T cell receptor "inside-out" pathway via signaling module SKAP1RapL regulates $T$ cell motility and interactions in lymph nodes. Immunity. 2010;32(4):541-56

33. Ho DW-H, Kai AK-L, Ng IO-L. TCGA whole-transcriptome sequencing data reveals significantly dysregulated genes and signaling pathways in hepatocellular carcinoma. Front Med. 2015:9(3):322-30.

34. Fury W, Batliwalla F, Gregersen PK, Li W. Overlapping probabilities of top ranking gene lists, hypergeometric distribution, and stringency of gene selection criterion. In: 2006 international conference of the IEEE 
engineering in medicine and biology society: 30 Aug.-3 Sept. 2006; 2006. 5531-34.

35. Bursac Z, Gauss CH, Williams DK, Hosmer DW. Purposeful selection of variables in logistic regression. Source Code Biol Med. 2008;3(1):17.

36. Sawai Y, Tamura S, Fukui K, Ito N, Imanaka K, Saeki A, Sakuda S, Kiso S, Matsuzawa Y. Expression of ephrin-B1 in hepatocellular carcinoma: possible involvement in neovascularization. J Hepatol. 2003;39(6):991-6.

37. Wang H, Wu K, Sun Y, Li Y, Wu M, Qiao Q, Wei Y, Han Z-G, Cai B. STC2 is upregulated in hepatocellular carcinoma and promotes cell proliferation and migration in vitro. BMB Rep. 2012;45(11):629-34

38. Xia Z, Huang M, Zhu Q, Li Y, Ma Q, Wang Y, Chen X, Li J, Qiu L, Zhang $J$, et al. Cadherin related family member 2 acts as a tumor suppressor by inactivating AKT in human hepatocellular carcinoma. J Cancer. 2019;10(4):864-73.

39. Islam F, Gopalan V, Lam AK-Y. RETREG1 (FAM134B): a new player in human diseases: 15 years after the discovery in cancer. J Cell Physiol. 2018;233(6):4479-89.
40. Pinto R, Carvalho AS, Conze T, Magalhães A, Picco G, Burchell JM, Taylor-Papadimitriou J, Reis CA, Almeida R, Mandel U, et al. Identification of new cancer biomarkers based on aberrant mucin glycoforms by in situ proximity ligation. J Cell Mol Med. 2012;16(7):1474-84.

41. Li H, Zhu W, Zhang L, Lei H, Wu X, Guo L, Chen X, Wang Y, Tang H. The metabolic responses to hepatitis $B$ virus infection shed new light on pathogenesis and targets for treatment. Sci Rep. 2015;5:8421.

42. Rezaei Tavirani M, Rezaei Tavirani M, Zamanian Azodi M. ANXA2, PRKCE, and OXT are critical differentially genes in Nonalcoholic fatty liver disease. Gastroenterol Hepatol Bed Bench. 2019;12(2):131-7.

\section{Publisher's Note}

Springer Nature remains neutral with regard to jurisdictional claims in published maps and institutional affiliations.
Ready to submit your research? Choose BMC and benefit from:

- fast, convenient online submission

- thorough peer review by experienced researchers in your field

- rapid publication on acceptance

- support for research data, including large and complex data types

- gold Open Access which fosters wider collaboration and increased citations

- maximum visibility for your research: over $100 \mathrm{M}$ website views per year

At BMC, research is always in progress.

Learn more biomedcentral.com/submissions 